\title{
Enfermedad de Crohn y linfoma primario de intestino delgado: reporte de caso
}

\section{A Case Report of Crohn's Disease and Primary Small Bowel Lymphoma}

\author{
Gabriel Alonso Mosquera-Klinger. ${ }^{* *}$
}

\footnotetext{
1 Especialista en medicina interna, gastroenterología y endoscopia digestiva. Hospital Pablo Tobón Uribe, Medellín, Colombia.

*Correspondencia: gami8203@yahoo.com.

Fecha recibido: $\quad 29 / 01 / 18$

Fecha aceptado: $30 / 03 / 18$
}

\begin{abstract}
Resumen
Paciente de 57 años, con cuadro clínico de casi 1 año de evolución de dolor abdominal, distensión, en estudios endoscópicos con úlceras en íleon distal de aspecto inflamatorio y estenosis en región ileocecal, con hallazgos patológicos e imagenológicos compatibles con enfermedad de Crohn estenosante de íleon distal y elevación de marcadores de inflamación incluyendo calprotectina fecal (más de 20 veces del valor normal) se dejó en tratamiento médico óptimo incluyendo esteroides, inmunomodulador y terapia biológica con antagonista de factor de necrosis tumoral (anti-TNF), experimentó buena respuesta clínica inicial, pero en el control endoscópico y de imágenes hubo evidencia de lesión de aspecto tumoral en el íleon distal, por lo que se planteó el manejo quirúrgico, con el que se confirmó una lesión neoplásica de origen hematolinfoide (linfoma) en el íleon distal. Se hace reporte de caso y revisión de la literatura.
\end{abstract}

\section{Palabras clave}

Enfermedad de Crohn, enfermedad inmunoproliferativa del intestino delgado, obstrucción intestinal, imagen por resonancia magnética.

\begin{abstract}
This is a case report of Crohn's Disease with a primary small bowel lymphoma and a literature review of the topic. The 57 year-old patient had suffered from abdominal pain and distention for almost one year prior to diagnosis. Endoscopic studies had found inflamed ulcers in the distal ileum stenosis in the ileocecal region. Pathological and imaging findings were compatible with stenosing Crohn's disease of the distal ileum. Inflammation markers including fecal calprotectin (more than 20 times the normal value) were elevated. Optimal medical treatment including steroids, an immunomodulator, and biological therapy with tumor necrosis factor antagonist (AntiTNF) was administered, and patient's initial response was good. Nevertheless, endoscopic and imaging follow-ups found a lesion in the distal ileum that appeared to be a tumor. Surgery found a neoplastic hematolymphoid tumor in the distal ileum.
\end{abstract}

\section{Keywords}

Crohn's disease, immune-proliferative disease of the small intestine, intestinal obstruction, magnetic resonance imaging.

\section{INTRODUCCIÓN}

La enfermedad de Crohn (EC) está caracterizada por lesiones discontinuas e inflamación transmural, que puede afectar cualquier parte del tracto gastrointestinal. La loca- lización más común es la región ileocolónica y el intestino delgado exclusivamente, estas 2 ubicaciones constituyen casi el $70 \%$ de los casos $(1,2)$. Las formas de presentación clínica en estudios de otras latitudes son inflamatoria (en casi el $80 \%$ de los casos) y luego, en orden de frecuencia, 
estenosante y fistulizante (3). En un estudio realizado en el Hospital Pablo Tobón Uribe se identificó que casi un tercio de los pacientes se presentó con variedad estenosante, dato que puede tener relación con el diagnóstico tardío (4). No hay una única prueba de oro para el diagnóstico de la EC. El diagnóstico se confirma mediante una sumatoria de datos clínicos y hallazgos endoscópicos, imagenológicos e histológicos, y pruebas bioquímicas $(5,6)$. En el compromiso de íleon distal es fundamental la realización de una ileocolonoscopia, que es superior a otras estrategias (5).

Los pacientes con EC tienen más riesgo de desarrollar neoplasias en el tracto digestivo que la población general, especialmente adenocarcinoma y linfomas en el intestino delgado. El problema radica en que los linfomas primarios en el intestino delgado son difíciles de diagnosticar en el contexto de EC ya que los síntomas suelen ser similares o superpuestos a la actividad inflamatoria.

Se presenta el caso de un paciente con diagnóstico de EC establecido por hallazgos clínicos, imagenológicos, endoscópicos e histológicos, quien desarrolló un linfoma en el íleon distal.

\section{PRESENTACIÓN DE CASO CLÍNICO}

Paciente de 57 años cuya ocupación es la ganadería, previamente sano, refirió que desde noviembre de 2015 inició con dolor en hemiabdomen inferior y en la región lumbar baja; evaluado en atención primaria lo que consideró un dolor lumbar de origen mecánico y se puso tratamiento con meloxicam $15 \mathrm{mg} /$ día durante casi 2 meses. En febrero de 2016 se sintió peor por aparición de distensión y dolor abdominal de tipo cólico en hemiabdomen inferior, por lo cual se le realizaron estudios de resonancia magnética nuclear (RMN) de abdomen, en el que se encontró osteocondrosis lumbar T12 y L1, sin otros hallazgos anormales. En la colonoscopia total se describió colon normal, no se describió compromiso de la válvula ileocecal y se encontraron úlceras profundas recubiertas de fibrina en íleon distal (toma de biopsias); en el reporte de biopsias hubo inflamación crónica activa ulcerada, sin granulomas. En los exámenes serológicos resultaron anticuerpos frente al citoplasma de los neutrófilos (ANCA) negativos $y$ anticuerpos anti-Saccharomyces cerevisiae (ASCA) positivos, sin anemia. Se consideró probable ileítis ulcerada en relación con antiinflamatorios no esteroideos (AINE). Se recomendó suspender los AINE y se le dio tratamiento con mesalazina oral $3 \mathrm{~g} /$ día durante 1 mes. Hubo mejoría de distensión y del dolor abdominal por este período.

3 meses después el paciente refirió reaparición y aumento en intensidad de sus síntomas, asociados con diarreas con pintas de sangre, variable en cantidad, de 2 hasta 5 deposiciones al día. Por esta razón se evaluó por gastroenterología: en la revisión por sistemas negó pérdida de peso y diaforesis nocturna, no tenía síntomas extradigestivos. Se le dejó un antiparasitario de amplio espectro (ciclo de nitaxozanida) y se repitió la colonoscopia total en 2 meses; además, se le indicaron reactantes de fase aguda y calprotectina fecal. Los resultados de exámenes fueron los siguientes: calprotectina fecal marcadamente elevada: $2100 \mathrm{mg} / \mathrm{kg} /$ heces (valor de referencia [VR] hasta 50), hemograma normal, velocidad de sedimentación globular (VSG): 28 y proteína C reactiva (PCR): 1,58 (VR hasta 0,8; elevadas).

La colonoscopia de control se realizó el 10 de enero 2017 y se encontró úlcera circunferencial en válvula ileocecal con severo edema, eritema, congestión (estenosis inflamatoria severa), con úlceras en el íleon distal; se tomaron múltiples biopsias (Figura 1) y se hospitalizó con alta sospecha de EC estenosante. En las biopsias se evidenció mucosa ulcerada constituida por un estroma de tejido fibroconectivo vascularizado con marcados cambios inflamatorios, alteración arquitectural focal caracterizada por aplanamiento de vellosidades y borde en cepillo conservado con esfacelo. Se encontró un denso infiltrado linfoplasmocitario con agregados linfoides, criptas atróficas y pérdida de células caliciformes sin evidencia de parásitos, granulomas, ni cambios displásicos.

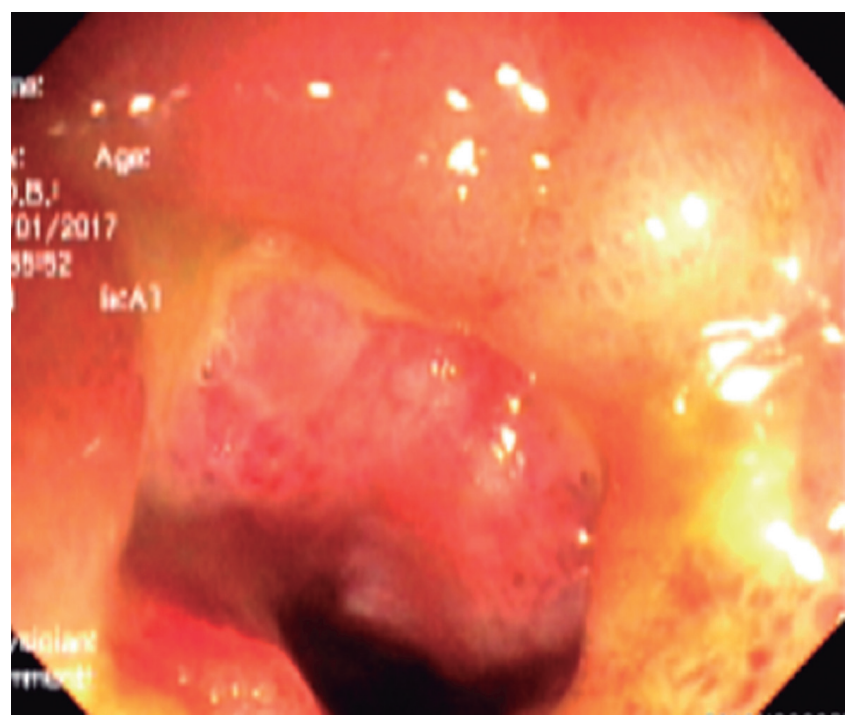

Figura 1. Severos cambios inflamatorios dados por edema, eritema, úlcera circunferencial en la válvula ileocecal e íleon distal.

Se solicitó enterografía por RMN (entero-RMN) en la que se encontró un marcado engrosamiento concéntrico, asimétrico e irregular de la submucosa de la pared de íleon distal con extensión a la válvula ileocecal. Se estimó el grosor de la pared de $1,4 \mathrm{~cm}$ y la extensión longitudinal de 7 $\mathrm{cm}$; también se encontró edema submucoso, restricción de difusión y realce poscontraste uniforme por cambios inflamatorios activos, estriación de grasa mesentérica (signo 
del cepillo), pequeñas adenopatías subcentimétricas no necróticas (hallazgos en relación con la actividad de EC, sin fibrosis) (Figura 2). Los estudios de tamizaje de infecciones resultaron negativos.

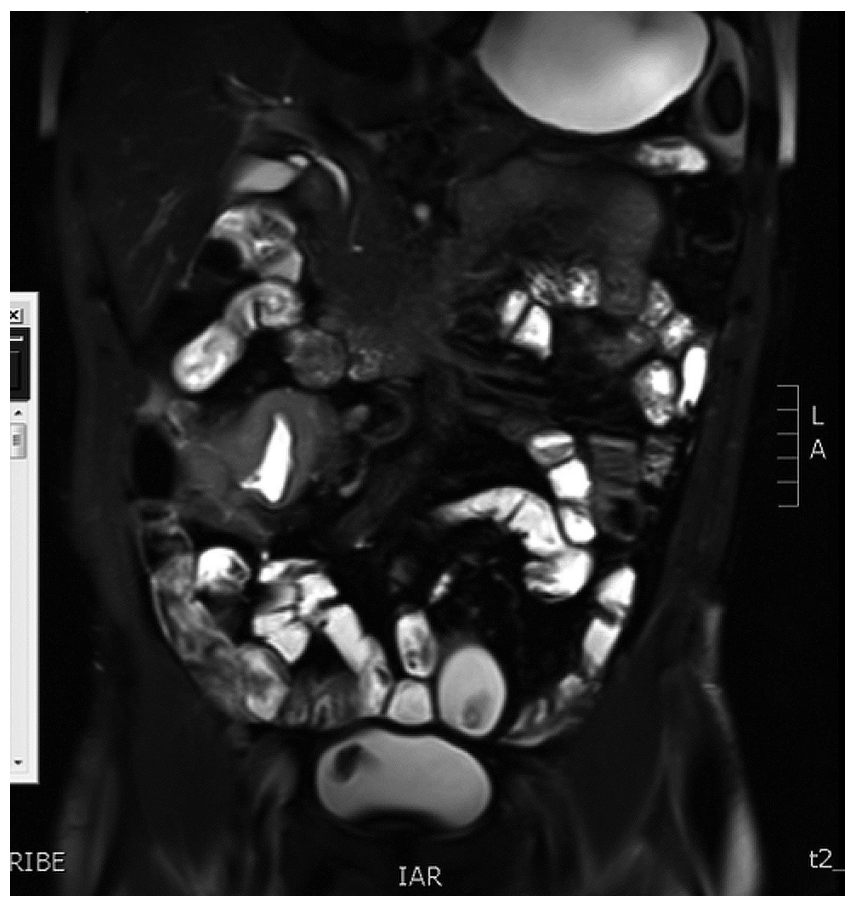

Figura 2. Enterorresonancia en la que se evidencia marcado engrosamiento en el íleon distal con áreas ulceradas y hallazgos de actividad de Crohn con compromiso inflamatorio-fibrótico.

Se le dio tratamiento con esteroides sistémicos (hidrocortisona $50 \mathrm{mg}$ cada 6 horas), azatioprina a $2 \mathrm{mg} / \mathrm{kg} /$ día, anti-
TNF (infliximab) en dosis de $5 \mathrm{mg} / \mathrm{kg} /$ dosis (en esquema de inducción: semanas 0,2 y 6 ). Hubo muy buena respuesta clínica dada por la mejoría del dolor abdominal, distensión y diarrea. Hubo descenso de reactantes de fase aguda; se le dio egreso y evaluación ambulatoria por gastroenterología.

4 meses después refirió reaparición del dolor abdominal y distensión; en el examen físico se palpó una masa dolorosa a nivel de la fosa ilíaca derecha, por lo cual se repitió una colonoscopia total en la que se encontró mayor engrosamiento en el íleon distal y úlcera circunferencial (Figura 3 A), y en la tomografía axial computarizada (TAC) de abdomen contrastado se reportó lesión pseudotumoral en el íleon distal hasta la válvula ileocecal (Figura 3 B); se programó para resección en cirugía. Se describió una lesión de aspecto tumoral, por lo que se realizó ilectomía y hemicolectomía derecha resecando casi $40 \mathrm{~cm}$ (Figura 4), con anastomosis ileotransversa. En patología se encontró neoplasia de estirpe hematolinfoide (linfoma B difuso de células grandes), lesión de $8 \mathrm{~cm}$, compromiso desde mucosa hasta serosa, bordes de resección libres de tumor, sin invasión vascular ni perineural; y ganglios negativos (Figura 5 A, B y C).

Se evaluó de forma ambulatoria por hematología que le complementó estudios de extensión con TAC de cuello y tórax, en los que se descartó compromiso nodal; además, se realizó aspirado de médula ósea, sin evidencia de compromiso a este nivel.

\section{DISCUSIÓN}

El paciente debutó con síntomas osteomusculares asociados con dolor abdominal y diarrea; con hallazgos seroló-
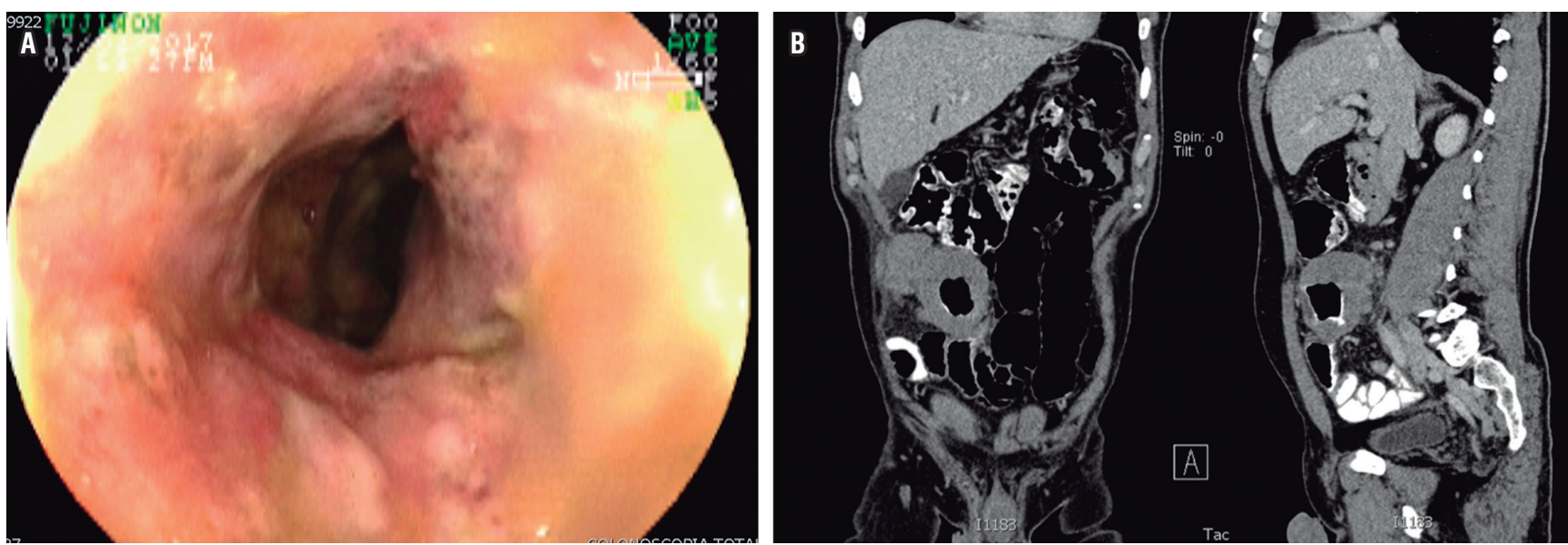

Figura 3. A. Mayor engrosamiento, además de severos cambios inflamatorios con ulceración circunferencial en la válvula ileocecal e íleon distal (4 meses después). B. TAC de abdomen contrastado, corte coronal en el que se identifica una lesión de aspecto tumoral en el íleon distal. 


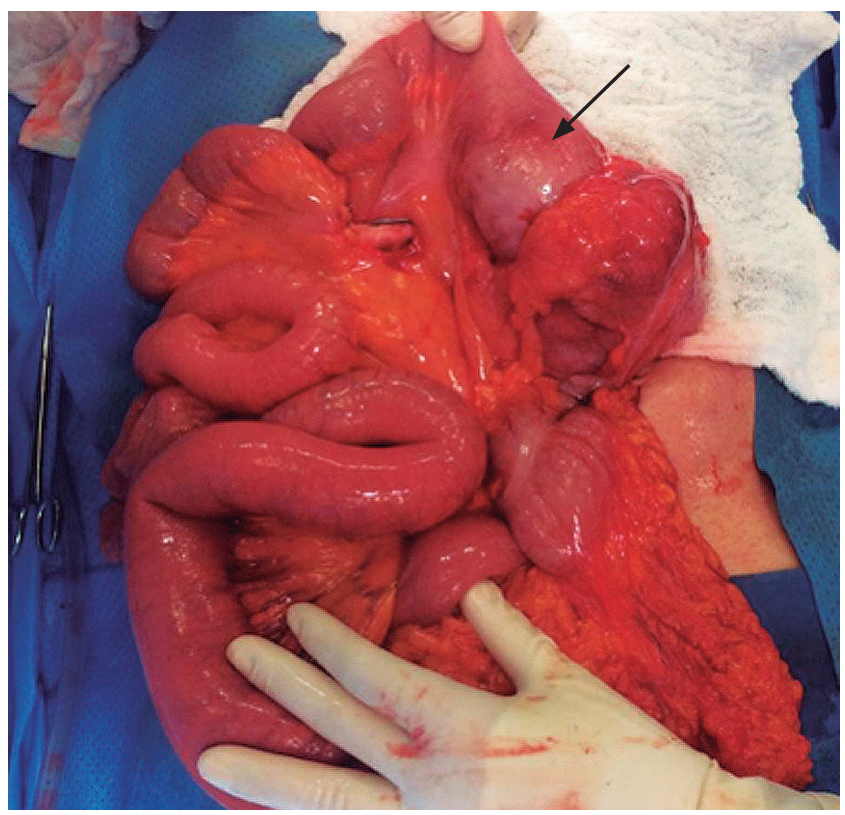

Figura 4. Foto durante la laparotomía en la que se identifica una lesión de aspecto tumoral. Se realizó ilectomía y hemicolectomía derecha.

gicos, endoscópicos e histológicos altamente sugestivos de EC con compromiso ileocecal, con un fenotipo estenosante, por lo cual requirió tratamiento con terapia biológica anti-TNF e inmunomodulador (azatioprina). Con el tratamiento hubo una notable mejoría clínica, pero empeoramiento progresivo de los hallazgos endoscópicos y de imágenes. En el seguimiento meses después hubo evidencia de masa palpable en la fosa ilíaca derecha, por lo cual se decidió llevar a resección quirúrgica programada (por vía laparoscópica) y en la pieza quirúrgica se determinó un linfoma primario del intestino delgado.
Las neoplasias del intestino delgado son raras, la incidencia global es generalmente menor de 1/100 000 (7). En Estados Unidos, el cáncer de intestino delgado representa $0,4 \%$ del total de neoplasias y el 0,2\% de muertes por cáncer $(7,8)$. El linfoma primario de intestino delgado es aún más inusual.

Los pacientes con EC tienen más riesgo de desarrollar cáncer colorrectal (especialmente adenocarcinomas) (9) y linfomas en el intestino delgado (especialmente linfoma no Hodgkin en íleon terminal) $(10,11)$, pero también hay datos sobre el aumento de la incidencia del adenocarcinoma de intestino delgado en EC $(12,13)$. El linfoma primario en el intestino delgado no es fácil de diagnosticar en EC ya que los síntomas pueden ser similares o superpuestos a la actividad inflamatoria. Únicamente la presencia de masa o signos obstructivos orientarían a esta complicación, como en el caso presentado.

$\mathrm{Al}$ igual que en la colitis ulcerativa, el riesgo de cáncer en pacientes con EC se correlaciona con la edad (es más frecuente cuando el diagnóstico es en edades tempranas), la duración y extensión de la enfermedad, además de la presencia de lesiones precancerosas (como displasia). En estudios poblacionales de pacientes con EC en el intestino delgado se ha estimado que el riesgo relativo de carcinoma en el intestino delgado aumenta de 10 a 40 veces (13). El riesgo acumulado a los 10 años se estimó en 0,2 \% (intervalo de confianza [IC] $95 \%: 0,0-0,8)$ y en 25 años, $2,2 \%$ (IC $95 \%$ : 0,7-6,4) (14).

El tipo de linfoma más agresivo y al que más se le teme durante el tratamiento con inmunosupresores de pacientes con EII es el linfoma hepatoesplénico de células T (ya que suele ser mortal). Se han reportado menos de 30 casos en todo el mundo (15); la mayoría de estos hombres tenía una mediana entre 12 a 58 años y estaba usando esquemas de tratamiento combinados (anti-TNF e inmunomodulado-
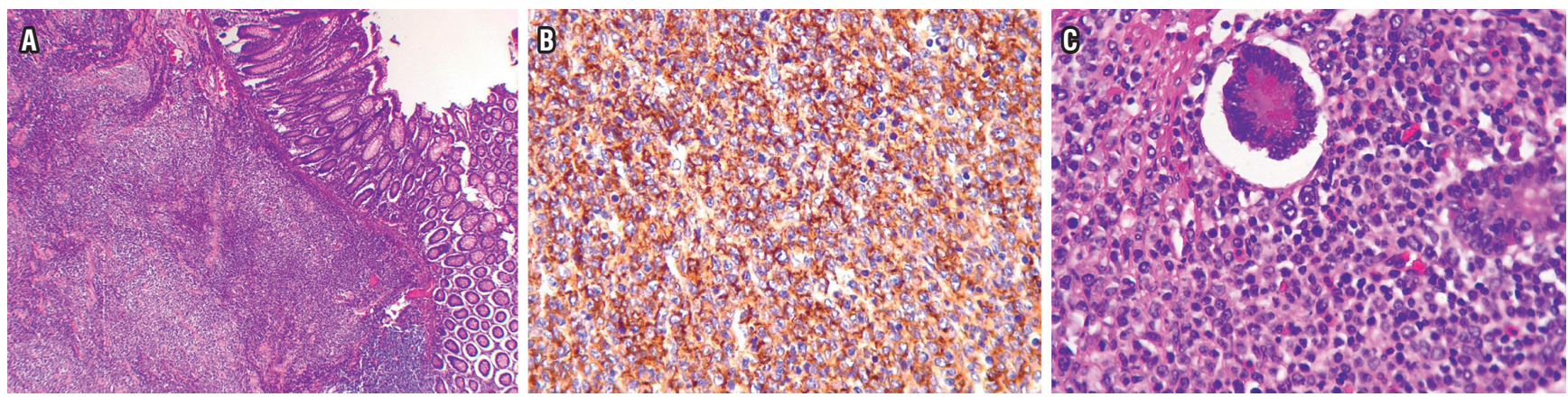

Figura 5. A. Foto en 4x. Neoplasia con patrón difuso que invade todo el espesor de la pared, incluida la mucosa, con ulceración focal. B. Inmunohistoquímica CD20, que muestra positividad fuerte y difusa para CD20 en la membrana de los linfocitos tumorales. C. Foto en 40x, mucosa a mayor aumento en la que se aprecian múltiples linfocitos neoplásicos de tamaños grandes y núcleos vesiculosos, los cuales son discohesivos y presentan abundantes figuras mitóticas, sin presencia de lesión linfoepitelial. 
res); aunque se han publicado varios casos de jóvenes en monoterapia con azatioprina (15-17).

En este caso presentado el diagnóstico de EC se basó en datos clínicos, exámenes, imágenes y hallazgos endoscópicos e histológicos siguiendo las recomendaciones recientes $(5,6)$. Por la evolución y los hallazgos endoscópicos (en las 2 primeras colonoscopias no había estenosis, ni masas) se determinó que desarrolló un linfoma en el intestino delgado a partir de la actividad de la EC. Lo llamativo de este caso es la rápida progresión a estenosis y la aparición de masa en tan poco tiempo en este paciente.

En un estudio previo se reportó una duración de EII al diagnóstico de linfoma de 3,1 años en promedio y la media de iniciación del inmunosupresor al diagnóstico de linfoma fue 20 meses (18). En este estudio se hizo seguimiento por 8 años a 782 pacientes con EII ( 238 de estos tenían inmunosupresión); de estos, se identificaron 4 casos con linfoma no Hodgkin (los 4 tenían inmunosupresores: 2 azatioprina, 1 metotrexato y 1 metotrexato y ciclosporina). El grupo de inmunosupresión tuvo 59 veces más riesgo de linfoma no Hodgkin que la población general (p: 0,0001) (18). Dicho comportamiento clínico denota un comportamiento muy agresivo del linfoma en este caso. Son múltiples las publicaciones que han descrito que no solo la actividad de la enfermedad aumenta el riesgo de linfoma, sino además el uso de inmunomoduladores $(15,19-22)$.

En este caso propusimos hacer seguimiento clínico y mediante entero-RMN de forma periódica.

La calprotectina es una proteína que se une al calcio y al zinc, presente de forma predominante en el citoplasma de las células que participan en la defensa de los patógenos, como los granulocitos neutrófilos, los monocitos y los macrófagos. La calprotectina muestra propiedades bacteriostáticas y fungistáticas in vitro, que subrayan su función en el ataque de patógenos. En los granulocitos neutrófilos, representa hasta el $60 \%$ de la proteína citosólica (23); esta se observa incrementada entre 5 a 40 veces en condiciones infecciosas e inflamatorias. Sus niveles son marcadamente elevados en heces de pacientes con EII (24). Tiene un excelente valor predictivo negativo en pacientes sintomáticos y su valor predictivo positivo es generalmente mejor que otros marcadores de inflamación utilizados, pero la limitación es que también puede elevarse en otras condiciones inflamatorias en el intestino delgado (5), aunque no hay evidencia de que este marcador se eleve de forma significativa en linfoma intestinal.

\section{CONCLUSIONES}

Las neoplasias del intestino delgado son infrecuentes y aún más raro el linfoma intestinal. El linfoma primario del intestino delgado se puede incrementar en el contexto de enfer- medades inflamatorias que comprometan dicha área; se ha descrito que la EC podría incrementar el riesgo de forma significativa, aún más en el escenario de la EC con fenotipo estenosante que requiera terapias inmunosupresoras para controlar la enfermedad.

\section{Agradecimientos}

Al Dr. Gabriel Varela Aguirre, patólogo-oncólogo del Hospital Pablo Tobón Uribe, Medellín, Colombia, por su valiosa colaboración y por las imágenes de la histopatología del caso.

\section{REFERENCIAS}

1. Garrido E, López A. Ileítis terminal: Diagnóstico diferencial. En: Marín-Jiménez I, Menchén-Viso L, Gomollón-García F (editores). Diagnóstico diferencial de la enfermedad inflamatoria intestinal. Elsevier; 2012. p. 23-32.

2. Hani A, Mosquera-Klinger G, Leguizamo AM. Presentación clínica de la enfermedad de Crohn. En: Aponte D, Gil F, Reyes G (editores). Diagnóstico en enfermedad inflamatoria intestinal. 1. ${ }^{a}$ edición. Bogotá D. C.: Pulso ediciones S.L; 2014. p. 81-90.

3. Rieder F, Zimmermann EM, Remzi FH, Sandborn WJ. Crohn's disease complicated by strictures: a systematic review. Gut. 2013;62(7):1072-84. doi: 10.1136/ gutjnl-2012-304353.

4. Juliao F, Ruiz M, Flórez J, Donado J, Marín J, Arango C, et al. Fenotipo e historia natural de la enfermedad inflamatoria intestinal en un centro de referencia en Medellín-Colombia. Rev Colomb Gastroenterol. 2010;25(3):240-51.

5. Gomollón F, Dignass A, Annese V, Tilg H, Van Assche G, Lindsay JO, et al. 3rd European Evidence-based Consensus on the Diagnosis and Management of Crohn's Disease 2016: Part 1: Diagnosis and Medical Management. J Crohns Colitis. 2017;11(1):3-25. doi: 10.1093/ecco-jcc/jjw168.

6. Yamamoto-Furusho JK, Bosques-Padilla F, de-Paula J, Galiano MT, Ibañez P, Juliao F, et al. Diagnosis and treatment of inflammatory bowel disease: First Latin American Consensus of the Pan American Crohn's and Colitis Organisation. Rev Gastroenterol Mex. 2017;82(1):46-84. doi: 10.1016/j.rgmx.2016.07.003.

7. Schottenfeld D, Beebe-Dimmer JL, Vigneau FD. The epidemiology and pathogenesis of neoplasia in the small intestine. Ann Epidemiol. 2009;19(1):58-69. doi: 10.1016/j.annepidem.2008.10.004.

8. Jemal A, Siegel R, Ward E, Hao Y, Xu J, Murray T, Thun MJ. Cancer statistics, 2008. CA Cancer J Clin. 2008;58(2):7196. doi: 10.3322/CA.2007.0010.

9. Hemminki K, Li X, Sundquist J, Sundquist K. Cancer risks in Crohn disease patients. Ann Oncol. 2009;20(3):574-80. doi: 10.1093/annonc/mdn595.

10. Holubar SD, Dozois EJ, Loftus EV Jr, Teh SH, Benavente LA, Harmsen WS, et al. Primary intestinal lymphoma in 
patients with inflammatory bowel disease: a descriptive series from the prebiologic therapy era. Inflamm Bowel Dis. 2011;17(7):1557-63. doi: 10.1002/ibd.21516.

11. Dawson MP, Cornes JS, Morson BC. Primary malignant lymphoid tumours of the intestinal tract. Report of 37 cases with a study of factors influencing prognosis. Br J Surg 1961;49:80-9.

12. Jess T, Loftus EV Jr, Velayos FS, Harmsen WS, Zinsmeister AR, Smyrk TC, et al. Risk of intestinal cancer in inflammatory bowel disease: a population-based study from olmsted county, Minnesota. Gastroenterology. 2006;130(4):103946. doi: $10.1053 /$ j.gastro.2005.12.037.

13. Friedman S. Cancer in Crohn's disease. Gastroenterol Clin North Am. 2006;35(3):621-39. doi: 10.1016/j. gtc.2006.07.008.

14. Jess T, Gamborg M, Matzen P, Munkholm P, Sørensen TI. Increased risk of intestinal cancer in Crohn's disease: a meta-analysis of population-based cohort studies. Am J Gastroenterol. 2005;100(12):2724-9. doi: 10.1111/j.15720241.2005.00287.x.

15. Siegel C. Risk of lymphoma in inflammatory bowel disease. Gastroenterol Hepatol. 2009;5(11):784-90.

16. Mittal S, Milner BJ, Johnston PW, Culligan DJ. A case of hepatosplenic gamma-delta T-cell lymphoma with a transient response to fludarabine and alemtuzumab. Eur J Haematol. 2006;76(6):531-4. doi: 10.1111/j.16000609.2006.00646.x.

17. NavarroJT, RiberaJM, MateJL, Granada I,JuncàJ,Batlle M, et al. Hepatosplenic T-gammadelta lymphoma in a patient with
Crohn's disease treated with azathioprine. Leuk Lymphoma. 2003;44(3):531-3.10.1080/1042819021000035662.

18. Farrell RJ, Ang Y, Kileen P, O’Briain DS, Kelleher D, Keeling PW, et al. Increased incidence of non-Hodgkin's lymphoma in inflammatory bowel disease patients on immunosuppressive therapy but overall risk is low. Gut. 2000;47(4):514-9.

19. Hecker R, Sheers R, Thomas D. Hodgkin's disease as a complication of Crohn's disease. Med J Aust. 1978;2(13):603.

20. Connell WR, Kamm MA, Dickson M, Balkwill AM, Ritchie JK, Lennard-Jones JE. Long-term neoplasia risk after azathioprine treatment in inflammatory bowel disease. Lancet. 1994;343(8908):1249-52.

21. Fraser AG, Orchard TR, Robinson EM, Jewell DP. Longterm risk of malignancy after treatment of inflammatory bowel disease with azathioprine. Aliment Pharmacol Ther. 2002;16(7):1225-32.

22. Kandiel A, Fraser AG, Korelitz BI, Brensinger C, Lewis JD. Increased risk of lymphoma among inflammatory bowel disease patients treated with azathioprine and 6-mercaptopurine. Gut. 2005;54(8):1121-5. doi: 10.1136/ gut.2004.049460.

23. Abraham BP, Kane S. Fecal markers: calprotectin and lactoferrin. Gastroenterol Clin North Am. 2012;41(2):483-95. doi: 10.1016/j.gtc.2012.01.007.

24. Konikoff MR, Denson LA. Role of fecal calprotectin as a biomarker of intestinal inflammation in inflammatory bowel disease. Inflamm Bowel Dis. 2006;12(6):524-34. 